This article was published in Progress in Organic Coatings, 77, 1741-1749, 2014

http://dx.doi.org/10.1016/j.porgcoat.2014.05.030

\title{
Stabilization of nano-TiO2 aqueous dispersions with poly(ethylene glycol)-b-poly(4-vinyl pyridine) block copolymer and their incorporation in photocatalytic acrylic varnishes
}

\author{
S. Monteiro ${ }^{a}$, A. Diasa , A.M. Mendes ${ }^{a}$, J.P. Mendes ${ }^{\text {b }}$, A.C. Serra ${ }^{a}$, , N. Rocha ${ }^{b}$, \\ J.F.J. Coelho ${ }^{\text {b }}$, F.D. Magalhães ${ }^{a, *}$ \\ a LEPABE, Faculdade de Engenharia, Universidade do Porto, rua Roberto Frias, 4200-465 Porto, Portugal \\ b CEMUC, Chemical Engineering Department, University of Coimbra, 3030-790 Coimbra, Portugal
}

\begin{abstract}
In this work, $\mathrm{TiO} 2$ nanoparticles were dispersed and stabilized in water using a novel type of dispersant based on tailor-made amphiphilic block copolymers of poly(ethylene glycol)-block-poly(4-vinyl pyridine) (mPEG- $b$-P4VP) prepared by atom transfer radical polymerization (ATRP). The performance of this new block copolymer as dispersant was compared to a polyelectrolyte dispersant commonly used for TiO2, sodium salt of polyacrylic acid (Na-PAA). The effect of dispersion technique and type and amount of dispersant on deagglomeration and stability of the $\mathrm{TiO} 2$ aqueous suspensions were studied. After incorporation in a standard waterborne acrylic varnish formulation, dry film transparency, photocatalytic activity, and nanoparticle cluster size were also evaluated. The results show that mPEG- $b$-P4VP copolymer with appropriate block lengths can have a better performance than Na-PAA in terms of aqueous dispersion stabilization and cluster size reduction in the acrylic matrix. This translates into higher film transparency and photocatalytic performance.
\end{abstract}

\section{Introduction}

Photocatalytic titanium dioxide ( $\mathrm{TiO}_{2}$ ) nanoparticles (nano-TiO2), normally anatase, are used in a wide range of applications, including self-cleaning, aircleaning, anti-microbial, anti-fouling, water treatment, and photovoltaic cells, on different matrixes and supports, such as plastics, ceramics and coating [1-4]. Due 
to its photocatalytic activity nano-TiO2 can be used to eliminate organic matter from a surface [4] [5]. This process is based on the formation of pairs of electrons and holes $\left(\mathrm{e}^{-} / \mathrm{h}^{+}\right)$uponabsorption of ultraviolet light corresponding to the band gap energy $(3.2 \mathrm{eV})$. The photo- generated positive holes react with water adsorbed at the surface, forming hydroxyl radicals. On the other hand, the electrons injected from the valence to the conduction band reduce adsorbed oxygen to superoxide. The hydroxyl radicals formed in this redox process can subsequently degrade oxidatively organic molecules at the surface $[5,6]$.

However, a significant difficulty is posed by the nanoparticles strong tendency to agglomerate, due to large specific surface area. This behaviour is generally associated with Van der Waals attraction forces, and can be compensated by electrostatic and steric stabilization $[7,8]$, or by specific surface treatments $[9,10]$, even though complete deagglomeration into primary particles may be difficult or even impossible [11]. In the particular case of applications that demand dispersion in aqueous medium, cluster size and stability can be critical factors with repercussion on the quality of the final product.

For nano-TiO2, a sodium salt of poly(acrylic acid) (Na-PAA) is often used as electrosteric stabilizer. It is known that this polyelectrolyte adsorbs at the surface by coordination between dissociated carboxyl groups and $\mathrm{Ti}^{4+}$ Lewis acid sites $[4,5]$.

The alternative use of amphiphilic block copolymers for aqueous stabilization of nanoparticulated metal oxides has been reported in literature [12,13], due to the possibility of selective anchoring of the hydrophobic block to the metal surface, whereas the hydrophilic, water-soluble, block creates steric and/or electro- static repulsive interactions. The use of 4 -vinyl pyridine (4VP) as the anchoring block, which is hydrophobic at $\mathrm{pH}$ above its $\mathrm{pKa}(\sim 4.5)$ [14], has been successfully used in the stabilization of nanoaggregates of superparamagnetic iron oxide nanoparticles [14]. The anchoring of pyridyl groups is achieved by formation of strong coordination complexes with the metal cations $[8,15]$. The advent of Controlled/"Living" Radical Polymerization (CLRP) brought unprecedented tools to synthesize polymers with controlled structures, chain-end functionalities, architecture and narrow molecular weight distribution [16-19]. Among the CLRP methods, Atom Transfer Radical Polymerization [20] is the most used method due to several intrinsic advantages, such as: easy procedures, commercial availability of the compounds, mild reaction conditions and, more recently, residual amounts of metal complexes [21-27].

In this paper, we report the use of an amphiphilic copolymer based on poly(ethylene glycol) and poly(4-vinyl pyridine) blocks (mPEG- $b$-P4VP) [28] for dispersing photocatalytic $\mathrm{TiO} 2$ nanoparticles in water, followed by its 
incorporation in an acrylic varnish formulation. Considering that the varnish is intended to have photocatalytic activity with minimal loss of transparency, only small amounts of nano-TiO2 were incorporated, and therefore reduction of cluster size is paramount to maximize efficiency. Well-defined copolymer blocks of different molecular weights were prepared by atom transfer radical polymerization (ATRP) to evaluate the effect of the block copolymer design on the dispersion efficacy and varnish performance.

\section{Experimental}

\subsection{Materials}

Photocatalytic nano-TiO2 used in this study was P25 from Evonik Industries (Germany). The reported average diameter of the primary particles is $21 \mathrm{~nm}$, and the specific surface area (BET) of the powder is $50 \pm 15 \mathrm{~m}^{2} / \mathrm{g}$. Sodium salt of polyacrylic acid (Na- PAA) with Mw $2100 \mathrm{~g} / \mathrm{mol}$ was purchased from SigmaAldrich. The aqueous acrylic resin Polidisp 7778 (ca. $46 \mathrm{wt} \%$ in solids), based on acrylic and methacrylic acid esters was supplied by Resiquimica (Mem-Martins, Portugal), as well as the other components of varnish formulation (Preventol BM5, Preventol A14-D, Additol VXW 6393, Additol VXW 6503, Polidisp 7778, Texanol, Rheolate 278).

Poly(ethylene glycol) methyl ether (mPEG) (mPEG113: Mw=5000 Da, and mPEG45: $\mathrm{Mw}=2500 \mathrm{Da}$; Sigma-Aldrich) was dried by azeotropic distillation from toluene. 2-Chloropropionyl chloride (CPC) (97\%; Sigma-Aldrich), $\mathrm{CuCl}_{2}$ $(99 \%+$ extra pure, anhydrous; Acros) were used as supplied. $\mathrm{Cu}(0)$ wire $(99 \%$; Acros) was activated with nitric acid, washed with acetone and dried before use. Isopropanol (IPA) (99.97\%; Fisher Chemical), diethyl ether (>99.8\%; SigmaAldrich), deuterated chloroform $(\mathrm{CDCl} 3) \quad(+1 \%$ tetramethylsilane (TMS); Eurisotop), sodium hydroxide (pellets QP; Panreac) and hydrochloric acid solution $(\mathrm{HCl})\left(37 \%\right.$; Aldrich) were used as received. Milli-Q water (Milli-Q ${ }^{\circledR}$, Millipore) was obtained by reverse osmosis. 4-vinylpyridine (4VP) (96\%; Fluka), triethylamine (TEA) (96\%; Sigma-Aldrich) and dichloromethane (DCM) (+99.6\%; Fisher Scientific) were dried and distilled under reduced pressure, prior to use. 4- Dimethylaminopyridine (DMAP) (99\%; ACROS) was recrystallized before use. Tris(2-dimethylaminoethyl)amine (Me6TREN) was synthesized according to procedures described in the literature [29]. The mPEG-Cl ATRP's macroinitiators were prepared through an adaptation of a reported methodology 
[30]. For Gel Permeation Chromatography (GPC), poly(methyl methacrylate) (PMMA) standards (Polymer Laboratories) (Acros, 99\%, 70 mesh) and high performance liquid chromatography (HPLC) DMF (HPLC grade; Panreac) were used as received.

\subsection{Synthesis of diblock copolymer dispersants}

mPEG-b-P4VP block copolymers were prepared by ATRP based on a previously reported methodology that used $\mathrm{Cu}(0)$ and $\left[\mathrm{CuCl}_{2}\right] /[\mathrm{Me} 6 \mathrm{TREN}]=1 / 1$ as the catalytic system [31]. In a typical procedure, a mixture of mPEG113-Cl (0.472 g, $0.09 \mathrm{mmol}), \mathrm{CuCl} 2$ (12.47 mg, $0.09 \mathrm{mmol})$, Me6TREN (21.36 mg, $0.09 \mathrm{mmol}$ ) and IPA $(3.11 \mathrm{~mL})$ was placed in a Schlenk reactor and immediately frozen in liquid nitrogen. Activated $\mathrm{Cu}(0)$ wire was placed in the reactor and the system was deoxygenated by three freeze-vacuum-thaw cycles and purged with nitrogen. $4 \mathrm{VP}(3.0 \mathrm{~mL}, 28 \mathrm{mmol})$ was then added to a Schlenk reaction vessel under a nitrogen atmosphere and the reaction mixture was immediately frozen in liquid nitrogen and deoxygenated by conducting two freeze-vacuum-thaw cycles and purged with nitrogen. The Schlenk reactor was placed in a pre- heated oil bath at $50{ }^{\circ} \mathrm{C}$ and left reacting under magnetic stirring. The reaction was stopped by opening the Schlenk reactor to air. The block copolymer was precipitated in cold diethyl ether and the solid dissolved in chloroform and passed through an alumina column. The solution was concentrated by rotary evaporation and the product recovered by precipitation in cold diethyl ether, followed by filtration. Final copper removal was achieved by re-dissolving the product in methanol and dialysis for $12 \mathrm{~h}$ (molecular weight cut-off $(\mathrm{MWCO})=1000 \mathrm{au}$ ). Finally, the crude product was obtained by precipitation in cold diethyl ether, filtered and dried, under vacuum, at $40^{\circ} \mathrm{C}$ for $48 \mathrm{~h}$.

Molecular weight distribution was determined by Gel Permeation Chromatography (GPC) using high performance size- exclusion chromatography (HPSEC), with refractive index (RI) (Knauer K-2301) detection. The column set consisted of a PL 10$\mu \mathrm{L}$ guard column (50 mm $7.5 \mathrm{~mm}$ ), followed by two MIXED-B PL columns (300 $\mathrm{mm} \quad 7.5 \mathrm{~mm}, 10 \mu \mathrm{L}$ ). DMF eluent containing $0.3 \%$ of $\mathrm{LiBr}$ was passed at a flow rate of $1 \mathrm{~mL} / \mathrm{min}$ and the columns were heated to $60^{\circ} \mathrm{C}$. Before injection, the samples were filtered through a polytetrafluoroethylene membrane with $0.2 \mu \mathrm{m}$ pore size. The system was calibrated against PMMA standards.

$400 \mathrm{MHz}{ }^{1} \mathrm{H}$ NMR spectra were recorded on a Bruker Avance III $400 \mathrm{MHz}$ spectrometer, with a 5-mm TXI triple resonance detection probe, in $\mathrm{CDCl} 3$ using tetramethylsilane (TMS) as an internal standard. 


\subsection{Preparation of nano-TiO2 aqueous dispersions}

The dispersant (mPEG- $b$-P4VP or Na-PAA) was first dissolved in water at a defined concentration. Then, nano-TiO2 $(1 \mathrm{wt} \%)$ was added and the $\mathrm{pH}$ adjusted to a value of 9 (typical $\mathrm{pH}$ for acrylic varnishes) using sodium hydroxide solution $0.2 \mathrm{~mol} \mathrm{~L}^{-1}$. Dispersant $\mathrm{mPEG} 45-b$-P4VP65 had to be dissolved at $\mathrm{pH}=$ 3.7 before $\mathrm{TiO} 2$ addition due to the high molecular weight of the P4VP block. Proto- nation of the basic pyridyl group at acidic $\mathrm{pH}$ induces solubilization. The final $\mathrm{pH}$ was then adjusted to 9 after adding the nanoparticles, as in the other cases.

Different techniques were used for dispersing the $\mathrm{TiO} 2$ nanoparticles, after $\mathrm{pH}$ correction: high-power ultrasound probe (UIP 1000hd by Hielsher Ultrasound Technology), rotor-stator high-shear mixer at $14000 \mathrm{rpm}$ (Ultra-Turrax T18 by IKA), and Cowles-type disperser at $2000 \mathrm{rpm}$ (Dispermat by VMA-Getzmann). At the end of the intended dispersion time, the $\mathrm{pH}$ value was measured again to confirm that it remained at 9 .

\subsection{Preparation of acrylic varnishes}

The varnish formulation was provided by Resiquimica and is shown in Table 1 . This is a previously validated standard formulation for waterborne acrylic varnishes. For preparation, the biocides, defoamer and wetting agent were first mixed in water using a helix impeller. The $\mathrm{pH}$ was adjusted to 9 by using an aqueous solution of $\mathrm{NaOH}(10 \mathrm{wt} \%)$. The acrylic resin was then added slowly and the mixture was homogenized for $10 \mathrm{~min}$. Finally, coalescent and thickener were added and stirring was maintained for $5 \mathrm{~min}$. For the photoactive formulations, $35 \mathrm{~g}$ of the previously prepared aqueous dispersion of nano-TiO2 was added before the acrylic resin. The total final mass was maintained by removing the corresponding amount of initial water.

\subsection{Particle size distribution}

After preparation, the dispersions were left to rest for $1 \mathrm{~h}$ and then a samplewas collected for particle size distribution (PSD) analysis on a Beckman Coulter LS230 light scattering system equipped with polarization intensity differential scattering (PIDS) analysis. The particle size distributions were computed by the equipment's software, based on Mie light scattering theory. 


\subsection{Dispersion stability}

Evaluation of nano-TiO2 dispersions stability along time was based on a reported methodology [13]. Each dispersion was left to rest for $1 \mathrm{~h}$ after its preparation, in a $50 \mathrm{~mL}$ flask. After that, a $3 \mu \mathrm{L}$ sample was carefully taken at certain liquid height with a micropipette and then diluted in $2.5 \mathrm{~mL}$ of distilled water. The absorbance of the diluted sample was measured on PU 8625 UV/VIS spectrophotometer at $320 \mathrm{~nm}$. This value was defined as the "time zero" measurement. Other collections were made at different time intervals during 15 days. Samples were always taken at the same liquid height. Three replicates were made for each dispersion formulation.

\subsection{Varnish transparency}

A film of varnish was applied on a glass slab with a wet thickness of $100 \mu \mathrm{m}$ with a blade applicator. The film was left to dry for $24 \mathrm{~h}$. The transmittance was measured at $700 \mathrm{~nm}$ on a Perkin Elmer Lambda 750 UV/VIS spectrophotometer, using a clean glass slab as reference.

\subsection{Photocatalytic activity}

The photocatalytic activity of the varnish was determined by degradation of blue methylene solution under UV light. A varnish film with a controlled wet thickness $(100 \mu \mathrm{m})$ was applied on the white portion of a Leneta 2 C opacity chart, and left to dry for at least $24 \mathrm{~h}$. Five drops of $150 \mathrm{mg} / \mathrm{L}$ blue methylene solution (50:50 ethanol/water) were spread over the varnish film and dried for $24 \mathrm{~h}$. After that, the blue methylene stain was irradiated under an average irradiance of 7 $\mathrm{W} \mathrm{m}^{-2}$ (Vilbert Lourmat lamp system) with a UV BLB $365 \mathrm{~nm}$ for $6 \mathrm{~h}$. The reflectance values were measured every $2 \mathrm{~h}$ at $600 \mathrm{~nm}$ using UV-3600 Shimadzu UV-VIS-NIR spectrophotometer.

\subsection{Transmission electron microscopy}

TEM images of nano-TiO2 dispersions were obtained on a Zeiss 902A/JEOL 1400, at IBMC - Institute for Molecular and Cell Biology (University of Porto). Dispersion samples were diluted in distilled water and a drop was placed on the TEM copper grip and left to dry. 


\subsection{Scanning electron microscopy}

Scanning electron microscopy (SEM) images were obtained using a JEOL JSM 35CNoran Voyager equipment, at CEMUP - Centro de Materiais da Universidade do Porto. Varnish film were applied on $1 \mathrm{~cm}^{2}$ glass slabs, dried for $24 \mathrm{~h}$ and then fractured in liquid nitrogen. Samples were coated with a gold/palladium alloy.

\section{Results and discussion}

\subsection{Selection of dispersion technique and conditions}

Different dispersion techniques were compared in order to identify the most effective in creating a well deagglomerated nano-TiO2 aqueous suspension. Sodium salt of polyacrylic acid (Na-PAA), conventionally used in industry for $\mathrm{TiO} 2$ aqueous dispersions was used as reference dispersant at this stage. Fig. 1 shows the volume particle size distributions (PSD) obtained, using Cowles disperser (30 min at $2000 \mathrm{rpm}$ ), rotor-stator mixer (30 min at $14000 \mathrm{rpm}$ ), and ultrasound probe ( $2 \mathrm{~min}$ at $50 \%$ amplitude). Using Cowles disperser the particles sizes were in three different ranges: 40-100 nm, 350-1000 nm and 1200-4000 nm. The larger agglomerates clearly dominate the distribution, showing that this technique is inefficient. The rotor-stator also provided unsatisfactory results, independently of the nanoparticles being added at once or gradually along the mixing time. The major difference observed in the Cowles's result is related to the shifting of the middle peak to the higher particle size, partly merging with the right-most peak. On the other hand, use of ultrasounds yielded a much better result, with a narrow peak centred at $0.1 \mathrm{~nm}$ and two much smaller ones in the 150-280 $\mathrm{nm}$ and 380-800 $\mathrm{nm}$ ranges. This result agrees with the work by Sato et al. [3], reporting better results with ultrasonication than with other techniques for dispersing nanosized $\mathrm{TiO} 2$ particles in water. The shock waves created by collapsing cavitations lead to high energy particle collisions and efficient deagglomeration. For comparison, the PSD obtained with ultrasonication and without Na-PAA dispersant is also shown. Very large agglomerates, with sizes above $1 \mu \mathrm{m}$ are formed, and only a small fraction of the dispersed mass lies in the $100 \mathrm{~nm}$ peak, showing the importance of the dispersant in stabilizing the nano- $\mathrm{TiO} 2$ particles in the aqueous medium.

The amplitude of sonotrode vibration was changed to evaluate its effect on cluster deagglomeration. Fig. 2 shows the particle size distribution of nano-TiO2 dispersions for amplitudes of 50\%, 80\% and 100\%. The results suggest that better 
deagglomeration can be obtained by using the maximum amplitude, with the peak centred at $600 \mathrm{~nm}$ almost disappearing.

For the selected ultrasonic vibration amplitude of $100 \%$, the ultrasonication time was increased up to $10 \mathrm{~min}$. Fig. 3 shows the slight improvements obtained in the PSD. The small peak at $600 \mathrm{~nm}$ is eliminated with 5 or 10 min sonication. However, it was observed that it appears again after a few hours resting. This observation, combined with the fact that these sonication times cause a significant increase in the liquid temperature, demanding cooling in an ice bath, lead to the decision to keep ultrasonication time at $2 \mathrm{~min}$. Also, a special attention was given to the time between dispersion preparation and PSD analysis. As shown in Fig. 4, agglomeration tends to become more significant along time due to limited dispersion stability. One-hour rest time after ultrasonication was carefully kept for all PSD measurements presented in this work.

\subsection{Block copolymer synthesis and characterization}

The use of an ATRP methodology to prepare the mPEG-P4VP block copolymers allowed obtaining block copolymers of con- trolled molecular weight and compositions. Table 2 presents the average molecular weight determined by NMR and GPC analysis and the polydispersity obtained from GPC analysis. The success of the adopted methodology is evidenced by the narrow molecular weight distributions and by the ease in controlling the molecular design.

Fig. 5 provides the NMR data spectrum obtained for the mPEG113-b-P4VP25-Cl block copolymers, where it is possible to identify the different peaks for each polymeric segment. Since the molecularweight of the mPEG segment (used as the ATRPmacroinitiator) is well known, and considering the narrow polydispersities obtained through the ATRP methodology, the P4VP chain length can be accurately determined through the integral ratio of P4VP to mPEG peaks in the NMR spectra. This value was, therefore, assumed as the P4VP chain length throughout the study.

GPC traces of the mPEG-b-P4VP block copolymers prepared from the mPEG45-

$\mathrm{Cl}$ macroinitiators are presented in Fig. 6. The complete shifting towards lower retention volumes for higher molecular weight block copolymers are a clear indication of the success of the adopted methodology, since higher P4VP to mPEG ratios correspond to block copolymers with higher molecular weights and with very narrow distributions.

Two different functionalized mPEGs were used as macroinitiators for the ATRP of $4 \mathrm{VP}$, a short segment of $2000 \mathrm{~g} / \mathrm{mol}(\mathrm{DP}=45)$ and a longer segment of 5000 $\mathrm{g} / \mathrm{mol}(\mathrm{DP}=113)$, in order to evaluate the influence of the hydrophilic of segment 
in the block copolymer. Then, the segment size of P4VP was also changed to determine the influence of the balance between the hydrophilic segment and the nano-TiO2 anchoring segment on the performance of the new dispersant. The influence of the block copolymer molecular weight, maintaining similar mPEG to P4VP molar ratios, can be provided by comparison of performance of block copolymers mPEG113-b- P4VP25 and mPEG113-b-P4VP8.

These block copolymers were tested as alternative dispersants to Na-PAA, using the methodology established for nano-TiO2 dispersion with Na-PAA. mPEG45 and mPEG113 homopolymers were also tested, in order to evaluate the performance of the hydrophilic chains alone.

\subsection{Particle size distribution}

For the selected dispersion conditions - ultrasonication at $100 \%$ amplitude for 2 min, the performance of MPEG- $b$-P4VP amphiphilic block copolymers was evaluated in terms of particle size distribution measured $1 \mathrm{~h}$ after dispersion. Fig. 7a shows the results obtained for mPEG113, mPEG113- $b$-P4VP8 and mPEG113- $b$-P4VP25. mPEG113 homopolymer was found to be unable to provide good stabilization of $\mathrm{TiO} 2$ nanoparticles, showing a very large fraction of agglomerates with sizes higher than $1 \mu \mathrm{m}$. Peng et al. had already shown that PEG is not effective for these systems at basic $\mathrm{pH}$ [4]. On the other hand, with the mPEG- $b$-P4VP copolymers the distributions show improving deagglomeration as the P4VP chain length increases. With these amphiphilic chains, formation of a self-assembled protective barrier at the particle surface is expected to occur, with the 4VP units strongly coordinated with the metal, and the mPEG blocks oriented towards the aqueous phase. This micellar conformation provides a more effective steric stabilization than mPEGalone, since an anchoring block is used. Nonetheless, the PSD for mPEG113- $b$-P4VP25 shows significantly more agglomerates than the PSD obtained with Na-PAA polyelectrolyte under the same conditions (see Fig. 2).

Fig. $7 \mathrm{~b}$ depicts the results for mPEG45, mPEG45- $b$-P4VP13, mPEG45- $b$-P4VP36 and mPEG45- $b$-P4VP65. Again, mPEG45 homopolymer showed a poor dispersant performance, even though the PSD shows smaller agglomerates above $1 \mu \mathrm{m}$ than for mPEG113. The mPEG45- $b$-P4VP13 copolymer provided a better dispersion, similar to that obtained with mPEG113- $b$-P4VP25. One should note that the two copolymers have similar ratios of number of mPEG to P4VP units: 3.5 and 4.5, respectively. On the other hand, the PSD for mPEG45- $b$ P4VP36 shows more agglomeration in the 300-500 nm range, and for mPEG45$b$-P4VP65 most of the material is in the form of agglomerates larger than $1 \mu \mathrm{m}$. 
These results show that increasing the ratio of P4VP to mPEG units is not beneficial to the dispersion of nano-TiO2. The hydrophobic (at $\mathrm{pH}$ 9) P4VP segments are expected to form aggregates with too strong hydrophobic character, which cannot be efficiently stabilized in an aqueous dispersion by relatively short hydrophilic mPEG blocks [28].

TEM images were obtained for dispersions prepared with $\mathrm{Na}$ - PAA and mPEG113-b-P4VP25 (Fig. 8). Both images show clusters of nano-TiO2 particles, but interestingly these are much larger for the dispersion stabilized with NaPAA. The dispersion prepared with the amphiphilic block copolymer shows better individualized particles forming agglomerates a few hundred nanometers in length. This result is apparently contradictory with the PSD measurements discussed above, where Na-PAA showed a more deagglomerated size distribution. It is important to note that the Beckman Coulter wet sample particle size analyzer uses a recirculation pump to move the particles through the sample cells and minimize agglomeration during analysis. TEM analysis, on the other hand, implies placing a drop of dispersion on the support grid and allow drying at rest. It is therefore suggested that even though Na-PAA provides smaller nano-TiO2 clusters, under no stirring conditions these tend to agglomerate faster than those stabilized with the mPEG-P4VP copolymer, i.e. dispersion stability at rest is worse with NaPAA. Another relevant aspect is that ionic stabilizers, such as Na-PAA, may lose effectiveness in dry medium. These issues will be analyzed further below.

\subsection{Dispersion stability}

Fig. 9 illustrates dispersion stability results during 15 days for dispersions without any stabilizer, and stabilized with mPEG113- $b$-P4VP25, mPEG45- $b$ P4VP13 (block copolymers with the most suitable PSD determined above), and Na-PAA. In Fig. 9, the zero value corresponds to $1 \mathrm{~h}$ resting after the sonication procedure. The samples prepared without dispersant presented very rapid sedimentation. After 15 days absorbance was near zero, with most nano-TiO2 deposited at the bottom of the sample holder. The samples containing dispersants showed much higher stability, but the one containing Na-PAA displayed lower absorbance throughout most measurements. This is consistent with the TEM observations of larger clustering in Na-PAA dispersions. mPEG113- $b$-P4VP25 presented higher absorbance among the samples tested.

For mPEG113- $b$-P4VP25, different weight ratios of dispersant to nano-particles $(\mathrm{r} / \mathrm{n})$ were tested. Fig. 10a shows that 1:4 ratio provided the better dispersion of nano-TiO2, followed by 1:1. It has been proposed that as dispersant concentration is increased, bridging flocculation may gain importance, 
counteracting the steric stabilization effect $[4,5]$. 1:6 and 1:10 ratios yielded similarly high agglomeration, indicating dispersant insufficiency. The corresponding dispersion stability measurements (Fig. 10b) show that 1:1 ratio had the most interesting results, followed closely by 1:4. The lower ratios, as expected, gave significantly poor stabilities, with an absorbance about $75 \%$ lower after 15 days.

\subsection{Film transparency}

Some of the dispersions previously studied were incorporated in waterborne acrylic varnishes, with $\mathrm{TiO} 2$ content equal to $0.4 \mathrm{wt} \%$ in relation to solids content. Transmittance at $700 \mathrm{~nm}$ was measured in a spectrophotometer to assess film transparency. From Fig. 11 it can be seen that when using Na-PAA as dispersant the transparency decreases about $37 \%$ in relation to the varnish without nanoTiO2. On the other hand, transparencies decrease only $26 \%$ with mPEG45- $b$ P4VP13 and 19\% with mPEG113- $b$-P4VP25. These lower transmittances, which are perceptible through visual inspection, suggest that nano- $\mathrm{TiO} 2$ is dispersed into smaller agglomerates within the acrylic matrix when mPEG-P4VP copolymers are used. This was confirmed by SEM imaging of the varnishes free surfaces (Fig. 12). It can be clearly seen that micron-sized agglomerates are present in the varnish with $\mathrm{TiO} 2 / \mathrm{Na}-\mathrm{PAA}$. The varnishes with the copolymer dispersants show $\mathrm{TiO} 2$ agglomerates in the tenths of micron. mPEG113- $b$-P4VP25, which had the highest transparency, presents an homogeneous distribution of very small agglomerates. SEM images taken from fracture surfaces after film breakage under liquid nitrogen, not shown here, revealed similar features across the thickness for all films.

The improved results obtained with mPEG-P4VP copolymers may not be directly related to only the better nano-TiO2 dispersion in water. During water evaporation, the polymeric particles coalesce to form a homogeneous film enveloping the filler. The dispersant used may play a role in compatibilizing the nanoparticles with the matrix, which mitigates the agglomeration in the dry coalesced film. Besides, it is known that ionic dispersants may not be effective in preserving dispersion stability during the film drying process [32]. Especially, if one considers the hydrophobic character of P4VP, the anchoring block may also play an important role in the compatibility with the acrylic matrix.

\subsection{Photocatalytic activity}

The varnish formulations were tested for degradation of blue methylene under 
UV light irradiation. The reflectance change history of the stained varnishes is shown in Fig. 13. The varnish containing nano-TiO2 stabilized with mPEG113b-P4VP25 showed the highest photocatalytic performance. After $6 \mathrm{~h}$ UV exposure, the increase in reflectance is $34 \%$ higher than for the Na-PAA stabilized sample.

\section{Conclusions}

The preparation of acrylic waterborne varnishes with photocatalytic activity provided by $\mathrm{TiO} 2$ nanoparticles was studied. Since dry film transparency is a key characteristic, the quality of nano-TiO2 deagglomeration throughout the polymer matrix is paramount. Ultrasonication revealed to be the most efficient to disperse the nanoparticles in water using conventional Na-PAA polyelectrolyte, when compared to mixing with rotor-stator or Cowles impeller.

Use of novel mPEG-b-P4VP block copolymers as dispersants, synthesized by ATRP with different molecular weights, yielded apparently more agglomerated particle size distributions in water than Na-PAA. However, dispersion absorbance versus time measurements indicated that the copolymers that gave better PSDs (mPEG113-b-P4VP25 and mPEG45-b-P4VP13) performed better than Na-PAA in terms of dispersion stability along time. TEM imaging of dried dispersions also indicated lower dry-state agglomeration with the block copolymers.

After incorporating the nanoparticle dispersions in an acrylic varnish formulation, lower loss in transparency and higher photocatalytic activity was obtained with the copolymers, especially with mPEG113-b-P4VP25, suggesting more effective cluster deagglomeration in the dry varnish film. SEM imaging confirmed that the films obtained from the dispersions prepared with MPEG113b-P4VP25 and mPEG45-b-P4VP13 contained much smaller clusters than those with Na-PAA.

The results suggest that the use of amphiphilic copolymers based on 4-vinyl pyridine as the anchoring block, and poly(ethylene glycol) as the steric stabilization block, is promising for incorporation of $\mathrm{TiO} 2$ nanoparticles in organic matrices prepared from waterborne dispersions, namely for photocatalytic applications.

\section{Acknowledgements}

Funding for this work was provided by FEDER, through Pro- grama Operacional Factores de Competitividade - COMPETE, and by national funding through FCT Fundaç ão para a Ciên- cia e a Tecnologia, in the framework of project Nanohybrid 
(PTDC/EQU-EPR/114354/2009), FCOMP 01-0102-FEDER 005365 and PTDC/EQU-EQU/115614/2009. Nuno Rocha acknowledges FCT-MCTES for his postdoctoral scholarship (SFRH/BPD/86352/2012).

\section{References}

[1] J.O. Carneiro, V. Teixeira, A. Portinha, A. Magalhães, P. Coutinho, C.J. Tavares,

R. Newton, Iron-doped photocatalytic TiO2 sputtered coatings on plastics for self-cleaning applications, Mater. Sci. Eng. B 138 (2007) 144-150.

[2] H. Lachheb, E. Puzenat, A. Houas, M. Ksibi, E. Elaloui, C. Guillard, J.-M. Herrmann, Photocatalytic degradation of various types of dyes (Alizarin $S$, Crocein Orange G, Methyl Red, Congo Red, Methylene Blue) in water by UVirradiated titania, Appl. Catal. B: Environ. 39 (2002) 75-90.

[3] K. Sato, J.-G. Li, H. Kamiya, T. Ishigaki, Ultrasonic dispersion of TiO2 nanoparti- cles in aqueous suspension, J. Am. Ceram. Soc. 91 (2008) 24812487.

[4] M.F. La Russa, S.A. Ruffolo, N. Rovella, C.M. Belfiore, A.M. Palermo, M.T. Guzzi,

G.M. Crisci, Multifunctional TiO2 coatings for cultural heritage, Prog. Org. Coat. 74 (2012) 186-191.

[5] A. Rachel, M. Sarakha, M. Subrahmanyam, P. Boule, Comparison of several titanium dioxides for the photocatalytic degradation of benzenesulfonic acids, Appl. Catal. B: Environ. 37 (2002) 293-300.

[6] J. Ângelo, L. Andrade, L.M. Madeira, A. Mendes, An overview of photocatalysis phenomena applied to NOx abatement, J. Environ. Manage. 129 (2013) 522539.

[7] S.H. Othman, S.A. Rashid, T.I.M. Ghazi, N. Abdullah, Dispersion and stabilization of photocatalytic $\mathrm{TiO} 2$ nanoparticles in aqueous suspension for coatings applications, J. Nanomater. 2012 (2012), 2-2.

[8] J. Jiang, G. Oberdörster, P. Biswas, Characterization of size, surface charge, and agglomeration state of nanoparticle dispersions for toxicological studies, J. Nanopart. Res. 11 (2009) 77-89.

[9] S. Pazokifard, S.M. Mirabedini, M. Esfandeh, M. Mohseni, Z. Ranjbar, Silane grafting of $\mathrm{TiO} 2$ nanoparticles: dispersibility and photoactivity in aqueous solutions, Surf. Interface Anal. 44 (2012) 41-47.

[10] J. Godnjavec, B. Znoj, N. Veronovski, P. Venturini, Polyhedral oligomeric silsesquioxanes as titanium dioxide surface modifiers for transparent acrylic UV blocking hybrid coating, Prog. Org. Coat. 74 (2012) 
654-659.

[11] C. Carneiro, R. Vieira, A.M. Mendes, F.D. Magalhães, Preparation and characterization of acrylic polymer nanocomposite films obtained from aqueous dispersions, J. Appl. Polym. Sci. 127 (2013) 2536-2543.

[12] M. Motornov, R. Sheparovych, R. Lupitskyy, E. MacWilliams, S. Minko, Responsive colloidal systems: reversible aggregation and fabrication of super- hydrophobic surfaces, J. Colloid Interface Sci. 310 (2007) 481-488.

[13] B. Peng, Y. Huang, L. Chai, G. Li, M. Cheng, X. Zhang, Influence of polymer dispersants on dispersion stability of nano-TiO2 aqueous suspension and its application in inner wall latex paint, J. Cent. South Univ. Technol. 14 (2007) 490-495.

[14] S. Mendrek, A. Mendrek, H.-J. Adler, A. Dworak, D. Kuckling, Synthesis and characterization of $\mathrm{pH}$ sensitive poly(glycidol)-b-poly(4vinylpyridine) block copolymers, J. Polym. Sci. A: Polym. Chem. 47 (2009) 1782-1794.

[15] N. Kang, B. Kang, H. Koh, M. Changez, J.-S. Lee, Block copolymers containing pyridine moieties: precise synthesis and applications, React. Funct. Polym. 69 (2009) 470-479.

[16] W.A. Braunecker, K. Matyjaszewski, Controlled/living radical polymerization: features, developments, and perspectives, Prog. Polym. Sci. 32 (2007) 93-146.

[17] J.F.J. Coelho, E.Y. Carvalho, D.S. Marques, A.V. Popov, P.M. Goncalves, M.H. Gil, Synthesis of poly(lauryl acrylate) by single-electron transfer/degenerative chain transfer living radical polymerization catalyzed by Na2S2O4 in water, Macromol. Chem. Phys. 208 (2007) 1218-1227.

[18] J.F.J. Coelho, E.Y. Carvalho, D.S. Marques, A.V. Popov, V. Percec, M.H. Gil, Influence of the isomeric structures of butyl acrylate on its single-electron transfer-degenerative chain transfer living radical polymerization in water catalyzed by Na2S2O4, J. Polym. Sci. A: Polym. Chem. 46 (2008) 6542-6551.

[19] J.F.J. Coelho, M. Carreira, P.M.O.F. Gonç alves, A.V. Popov, M.H. Gil, Processability and characterization of poly(vinyl chloride)-b-poly(n-butyl acrylate)-b- poly(vinyl chloride) prepared by living radical polymerization of vinyl chloride. Comparison with a flexible commercial resin formulation prepared with PVC and dioctyl phthalate, J. Vinyl Addit. Technol. 12 (2006) 156-165.

[20] T. Guliashvili, P.V. Mendonça, A.C. Serra, A.V. Popov, J.F.J. Coelho, Copper- mediated controlled/living radical polymerization in polar solvents: insights into some relevant mechanistic aspects, Chem. Eur. J. 18 (2012) 4607-4612. 
[21] W. Jakubowski, K. Matyjaszewski, Activator generated by electron transfer for atom transfer radical polymerization, Macromolecules 38 (2005) 4139-4146.

[22] K. Matyjaszewski, W. Jakubowski, K. Min, W. Tang, J. Huang, W.A. Braunecker, N.V. Tsarevsky, Diminishing catalyst concentration in atom transfer radical polymerization with reducing agents, Proc. Natl. Acad. Sci. U S A 103 (2006) 15309-15314.

[23] M. Rico, J. López, B. Montero, C. Ramírez, R. Bouza, Thermodynamic analysis of polymerization-induced phase separation of a polystyrene in epoxy/monoamine-diamine systems. Effect of monoamine-diamine proportion on the phase diagram, Eur. Polym. J. 47 (2011) 1676-1685.

[24] C.M.R. Abreu, P.V. Mendonç a, A.C. Serra, A.V. Popov, K. Matyjaszewski, T. Guliashvili, J.F.J. Coelho, Inorganic sulfites efficient reducing agents and supplemental activators for atom transfer radical polymerization, ACS Macro Lett. 1 (2012) 1308-1311.

[25] C.M.R. Abreu, A.C. Serra, A.V. Popov, K. Matyjaszewski, T. Guliashvili, J.F.J. Coelho, Ambient temperature rapid SARA ATRP of acrylates and methacrylates in alcohol-water solutions mediated by a mixed sulfite $/ \mathrm{Cu}(\mathrm{ii}) \mathrm{Br} 2$ catalytic system, Polym. Chem. 4 (2013) 5629-5636.

[26] R.A. Cordeiro, N. Rocha, J.P. Mendes, K. Matyjaszewski, T. Guliashvili, A.C. Serra,

J.F.J. Coelho, Synthesis of well-defined poly(2-(dimethylamino)ethyl methacrylate) under mild conditions and its co-polymers with cholesterol and PEG using Fe(0)/Cu(II) based SARA ATRP, Polym. Chem. 4 (2013) 3088-3097. [27] P.V. Mendonça, A.C. Serra, J.F.J. Coelho, A.V. Popov, T. Guliashvili, Ambient temperature rapid ATRP of methyl acrylate, methyl methacrylate and styrene in polar solvents with mixed transition metal catalyst system, Eur. Polym. J. 47 (2011) 1460-1466.

[28] N. Rocha, J. Mendes, L. Duraes, H. Maleki, A. Portugal, C.F.G.C. Geraldes, A. Serra, J. Coelho, Poly(ethylene glycol)-block-poly(4-vinyl pyridine) as a versatile block copolymer to prepare nanoaggregates of superparamagnetic iron oxide nanoparticles, J. Mater. Chem. B 2 (2014) $1565-1575$.

[29] M. Ciampolini, N. Nardi, Five-coordinated high-spin complexes of bivalent cobalt, nickel, and copper with tris(2-dimethylaminoethyl)amine, Inorg. Chem. 5 (1966) 41-44.

[30] K. Jankova, X. Chen, J. Kops, W. Batsberg, K. Jankova, X. Chen, J. Kops, W. Batsberg, Synthesis of amphiphilic PS-b-PEG-b-PS by atom transfer radical polymerization, Macromolecules 31 (1998) 538-541. 
[31] K.R.M. Vidts, F.E. Du Prez, Design of water-soluble block copolymers containing poly(4-vinylpyridine) by atom transfer radical polymerization, Eur. Polym. J. 42 (2006) 43-50.

[32] S. Farrokhpay, A review of polymeric dispersant stabilisation of titania pigment, Adv. Colloid Interface Sci. 151 (2009) 24-32. 


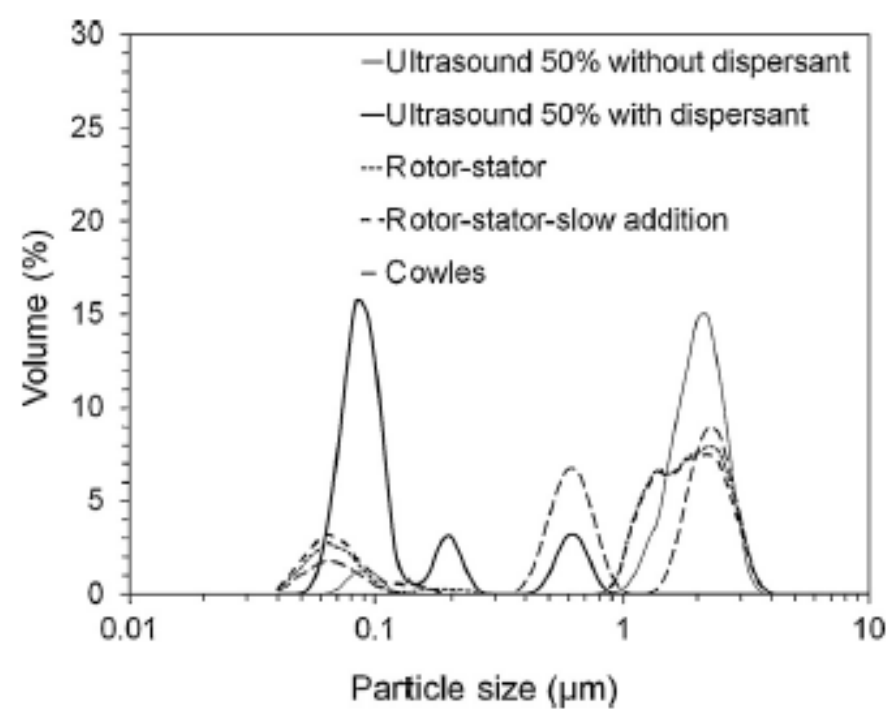

Fig. 1. Particle size distributions of nano-TiO2 aqueous dispersions $(10 \mathrm{mg}$ $\mathrm{mL}^{-1}, \mathrm{pH}=9$ ) prepared using different dispersion techniques. When dispersant (Na-PAA) was used, the ratio to nano-TiO2 was 1:1

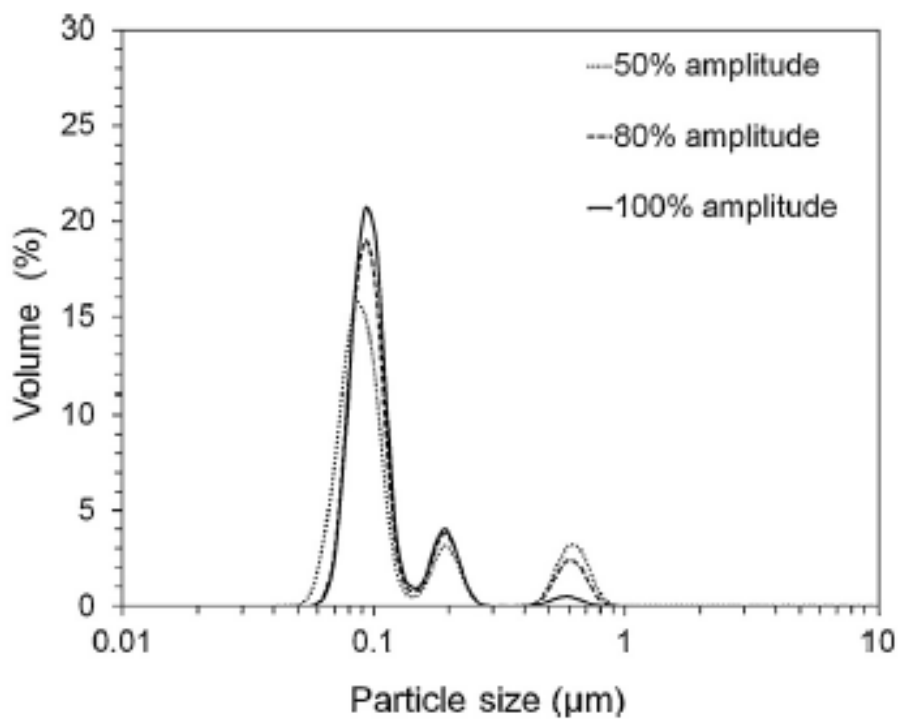

Fig. 2. Particle size distributions of nano-TiO2 aqueous dispersions stabilized with $1 \mathrm{wt} \% \mathrm{Na}-\mathrm{PAA}$, and prepared under different ultrasonic amplitudes during $2 \mathrm{~min}$. 


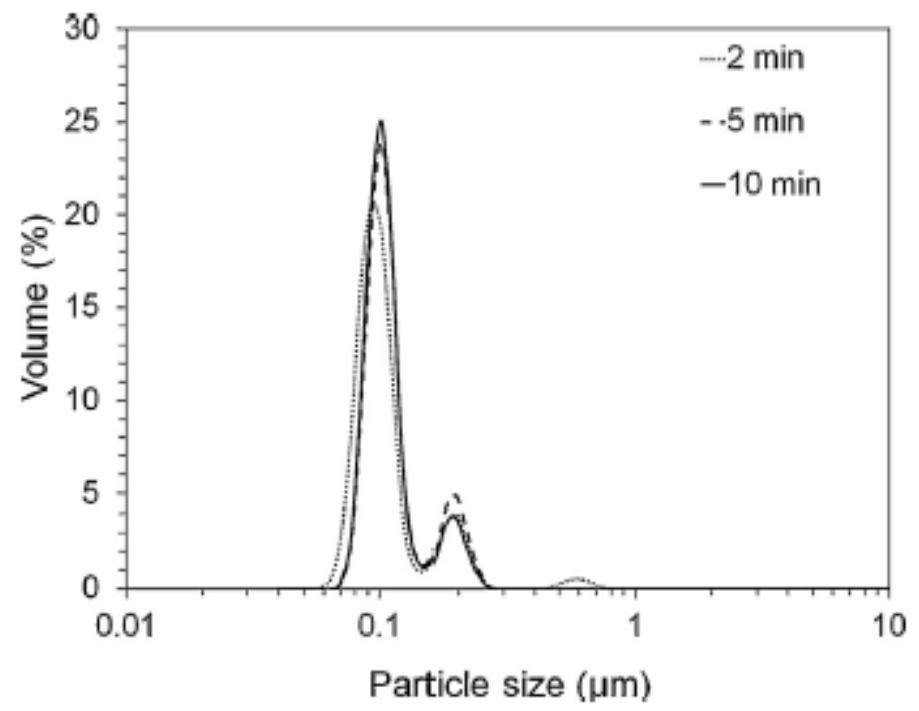

Fig. 3. Particle size distributions of nano-TiO2 aqueous dispersions stabilized with Na-PAA, and prepared under 100\% ultrasonic amplitude and different times

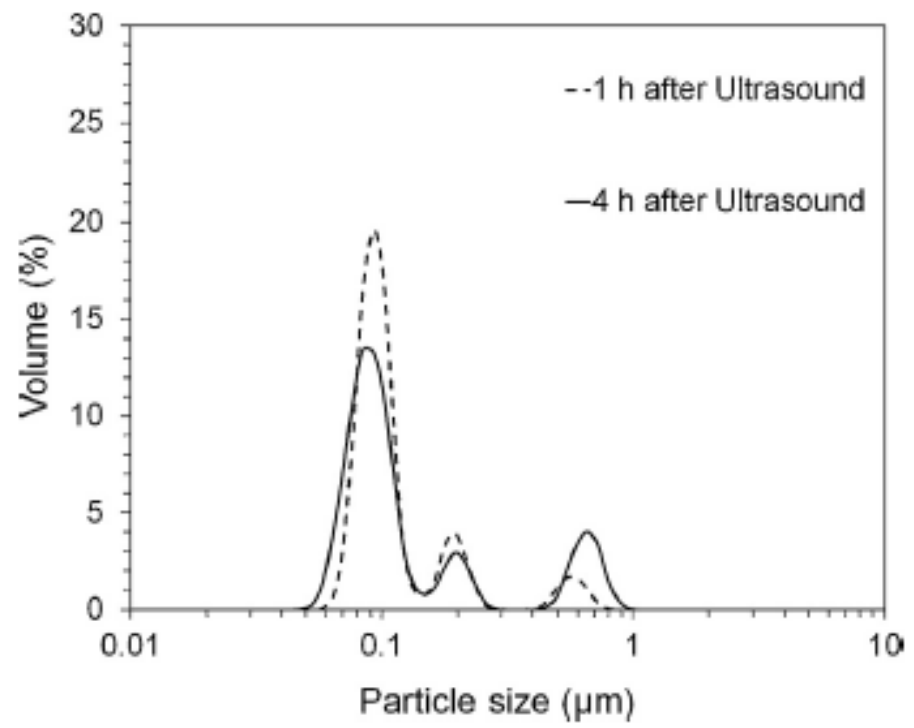

Fig. 4. Particle size distributions of nano-TiO2 aqueous dispersions stabilized with Na-PAA, one and four hours after the dispersion was prepared. 


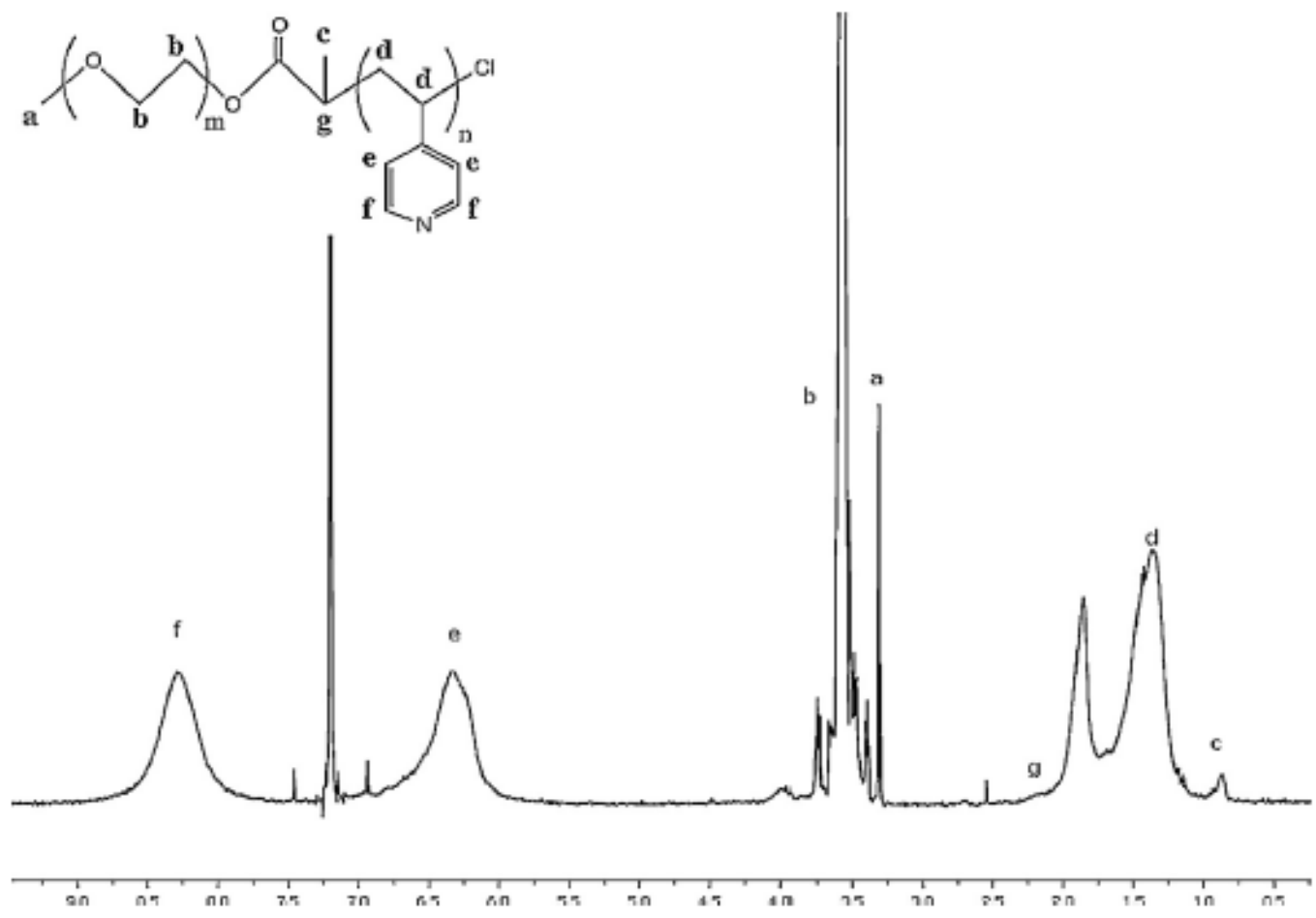

Fig. 5. ${ }^{1} \mathrm{H}$ NMR spectrum of mPEG113-b-P4VP25-Cl block copolymer. Their chemical structure and the proton identification scheme adopted for the NMR spectral assignments are also indicated.

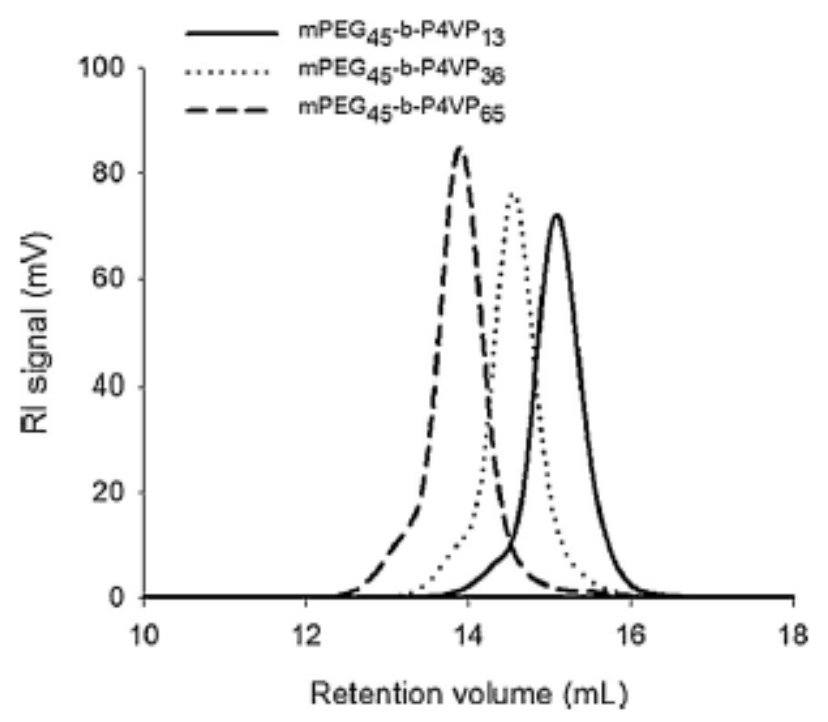

Fig. 6. GPC traces that were obtained for the block copolymers prepared from initiation of mPEG45-Cl 

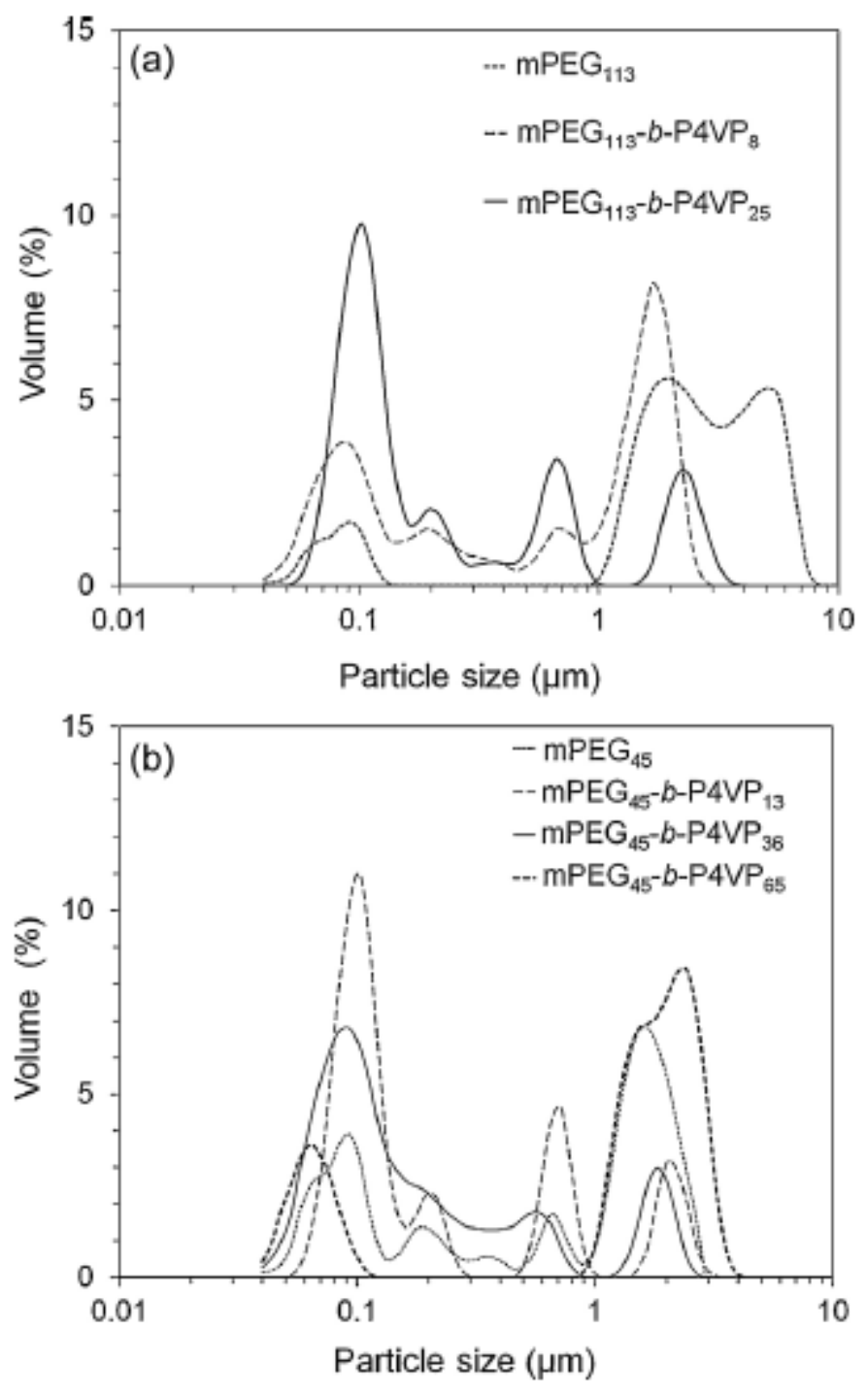

Fig. 7. Particle size distributions of nano-TiO2 aqueous dispersions $(10 \mathrm{mg}$ $\mathrm{mL}^{-1}, \mathrm{pH}=9$ ) prepared by ultrasonication ( $2 \mathrm{~min}$ at $100 \%$ amplitude) with dispersant/nano-TiO2 weight ratio 1:1. The dispersants used were: a) mPEG113, mPEG113- $b$-P4VP8 and mPEG113- $b$-P4VP25, and b) mPEG45, mPEG45- $b$-P4VP13, mPEG45- $b$-P4VP36 and mPEG45- $b$-P4VP65. 

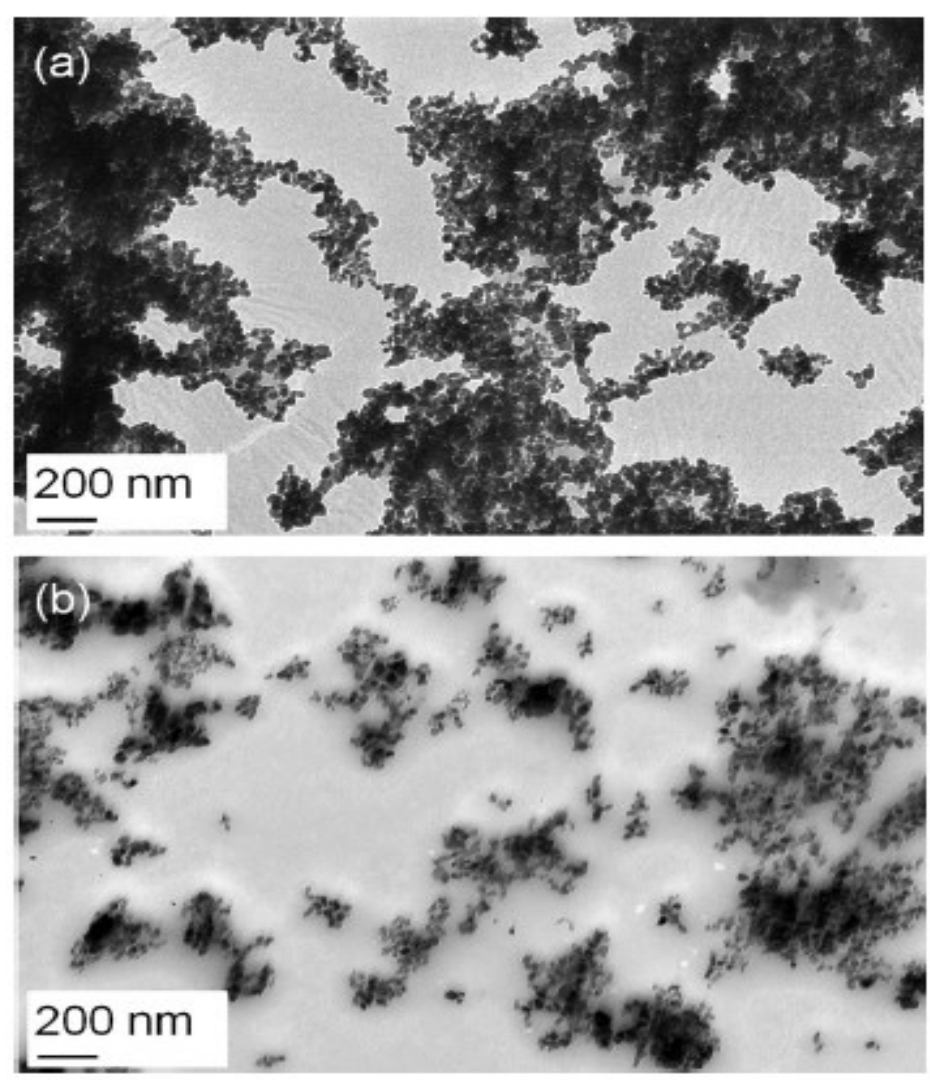

Fig. 8. TEM micrographs of $\mathrm{TiO} 2$ nanoparticles suspensions at $60000 \mathrm{x}$ magnification a) with Na-PAA and b) with mPEG45- $b$-P4VP13.

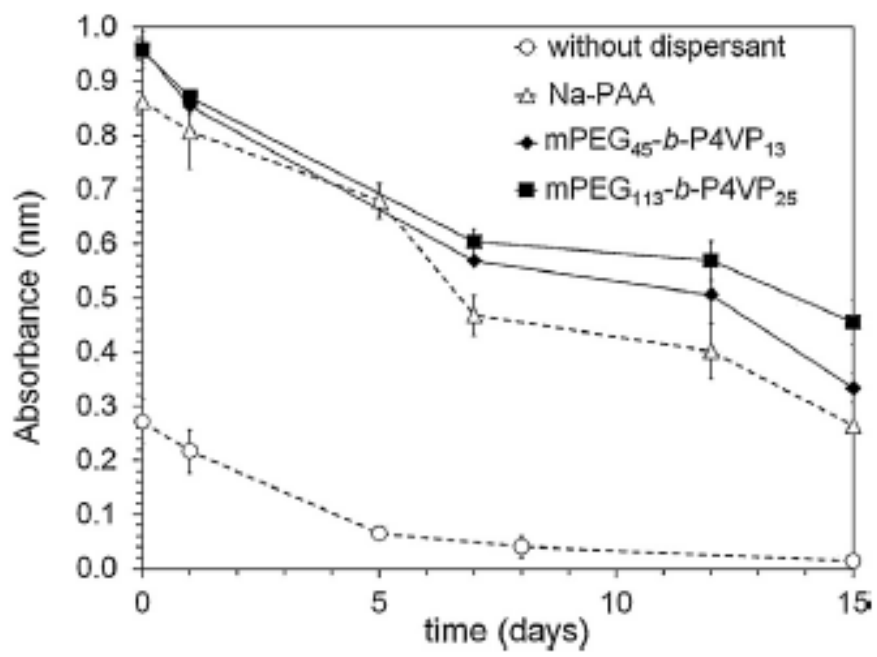

Fig. 9. Dispersion stability along time for nano-TiO2 aqueous dispersions with and without dispersant. Dispersions were prepared in the same conditions described in Fig. 7. The measurements are an average of three replicas; error bars represent standard deviations 

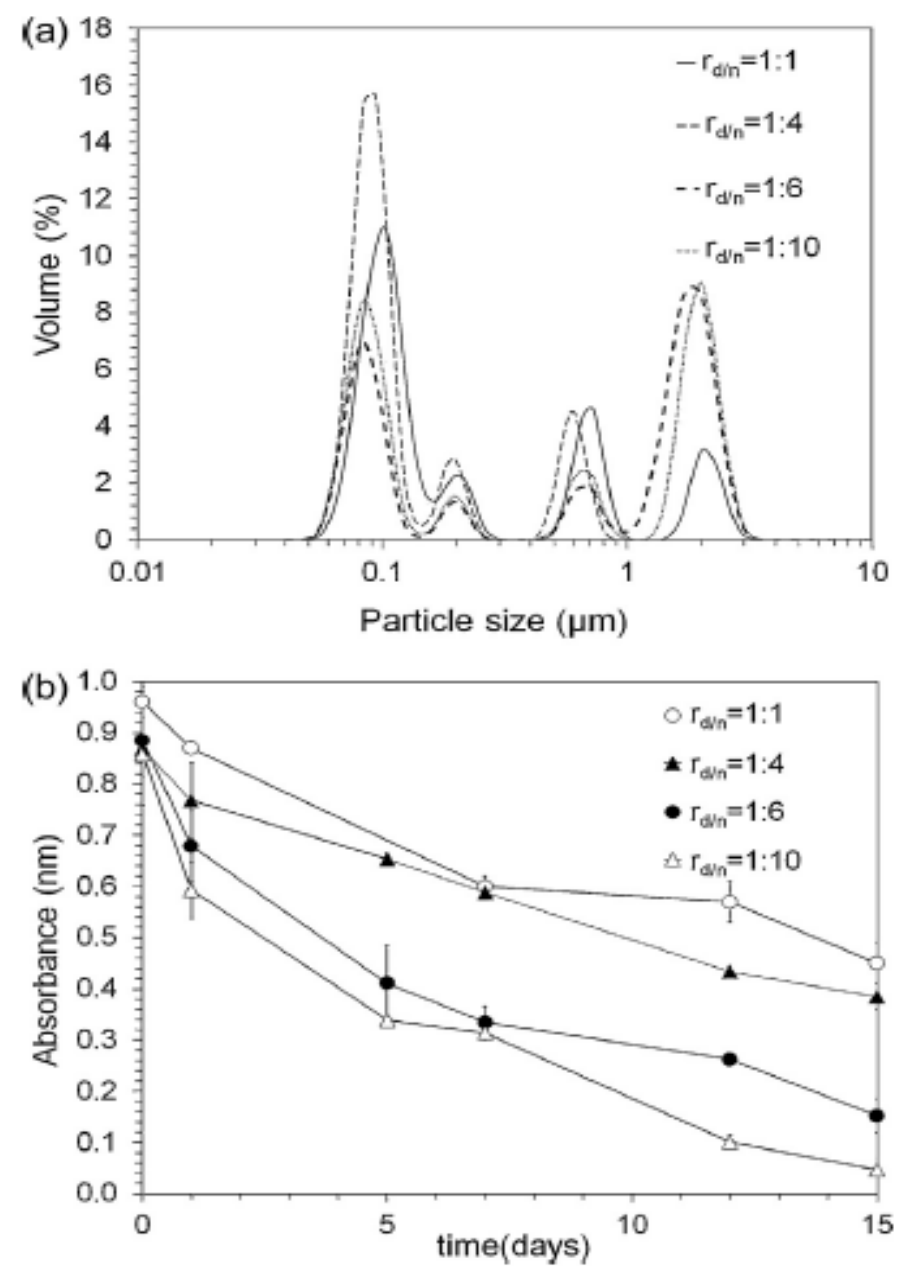

Fig. 10. Particle size distributions history of nano-TiO2 aqueous dispersions (a), and dispersion stability (b), for different dispersant/nano-TiO2 weight ratios: $r d / n=1: 1, r d / n=1: 4, r d / n=1: 6$ and $r d / n=1: 10$. The dispersant used was mPEG113- $b$-P4VP25

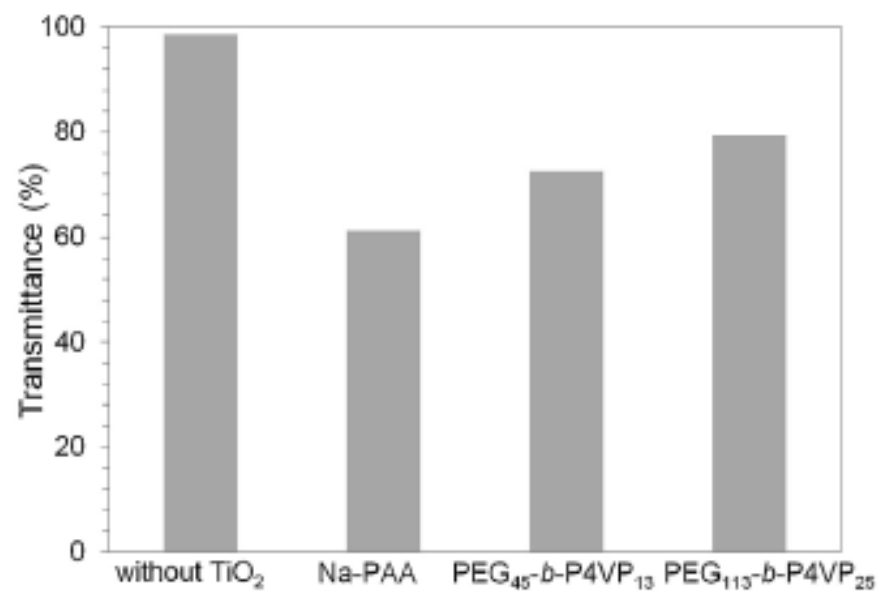

Fig. 11. Transmittance of different varnish films, with and without nano$\mathrm{TiO} 2$, at $700 \mathrm{~nm}$. Films contain $0.4 \mathrm{wt} \%$ of nano-TiO2 and the same amount of dispersant. 

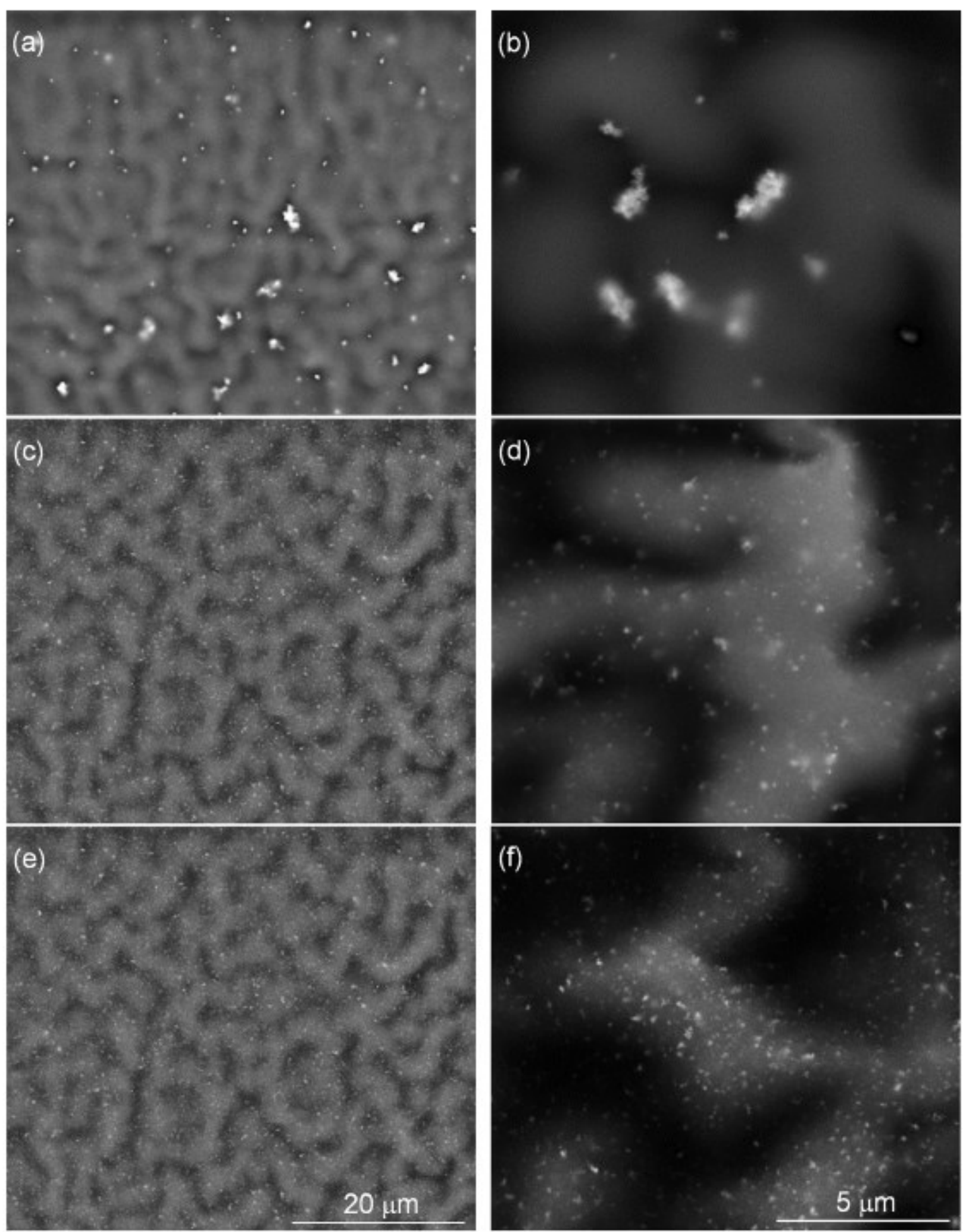

Fig. 12. SEM micrographs of surface of varnishes containing nano-TiO2 stabilized with different dispersants. (a) and (b) Na-PAA, (c) and (d) mPEG45- $b$-P4VP13, (e) and (f) mPEG113- $b$-P4VP25. Magnification is $5000 \times$ for images on the left column and $20000 \times$ for images on the right column 


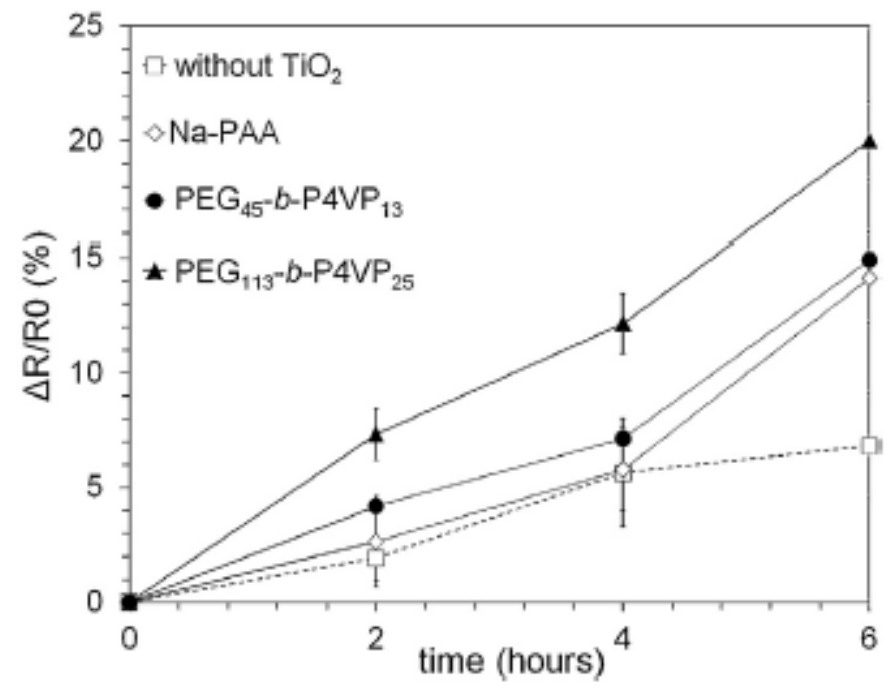

Fig. 13. Reflectance variation of different varnishes stained with blue methylene, with and without nano-TiO2, versus irradiation time. Samples contain $0.4 \mathrm{wt} \%$ nano- $\mathrm{TiO} 2$ and the same amount of dispersant, except for the one labelled "without $\mathrm{TiO} 2$ ".

Table 1

Formulation of acrylic varnish

\begin{tabular}{llc}
\hline Component & Function & Quantity (wt\%) \\
\hline Water & Coating medium & 41,20 \\
Preventol BM5 & Biocide & 0,20 \\
Preventol A14-D & Biocide & 0,70 \\
Additol VXW 6393 & Defoamer & 0,20 \\
Additol VXW 6503 & Wetting agent & 0,50 \\
NaOH 10\% solution & pH adjustment & 0,20 \\
Polidisp 7778 & Acrylic binder & 55,00 \\
Texanol & Coalescent & 1,50 \\
Rheolate 278 & Thickener & 0,50 \\
\hline
\end{tabular}

\section{Table 2}

$\bar{M} n$ and $\bar{M} w / \bar{M} n$ values determined by ${ }^{1} \mathrm{H}$ NMR and GPC for the mPEG- $b-$ P4VP block copolymer products.

\begin{tabular}{|c|c|c|c|}
\hline Block copolymer & $\begin{array}{l}\bar{M}_{\mathrm{a}} \mathrm{NMR} \\
(\mathrm{g} / \mathrm{mol})\end{array}$ & $\bar{M}_{\mathrm{n}} \mathrm{GPC}(\mathrm{g} / \mathrm{mol})$ & $\bar{M}_{w} / \bar{M}_{n}(\mathrm{GPC})$ \\
\hline $\mathrm{mPEG}_{45}-b-\mathrm{P}_{4} \mathrm{VP}_{13}$ & 3456 & 6955 & 1.12 \\
\hline $\mathrm{mPEG}_{45}-b-\mathrm{P} 4 \mathrm{VP}_{36}$ & 5875 & 11285 & 1,31 \\
\hline $\mathrm{mPEG}_{45}-b-\mathrm{P}_{4} \mathrm{VP}_{\bar{\xi}}$ & 8823 & 19790 & 1,16 \\
\hline $\mathrm{mPEG}_{113}-b-\mathrm{P} 4 \mathrm{VP}$ & 5931 & 13848 & 1,17 \\
\hline $\mathrm{mPEG}_{113}-$ b-P4VP 25 & 7719 & 16135 & 1.12 \\
\hline
\end{tabular}

